\title{
ПЕРЕХОДНЫЕ ПРОЦЕССЫ ПРИ КИПЕНИИ ЖИДКОСТИ НА ПОРИСТОЙ ПОВЕРХНОСТИ
}

\section{(Представил И. Эпик)}

В $\left[{ }^{1,2}\right]$ исследовались гистерезисные явления при кипении жидкости на тонкопористой поверхности. Были установлены закономерности существования гистерезисной петли кривой кипения в зависимости от параметров пористого покрытия.

В связи с наличием гистерезисных явлений в области пузырькового режима кипения, широко применяемого на практике, представляют ин-

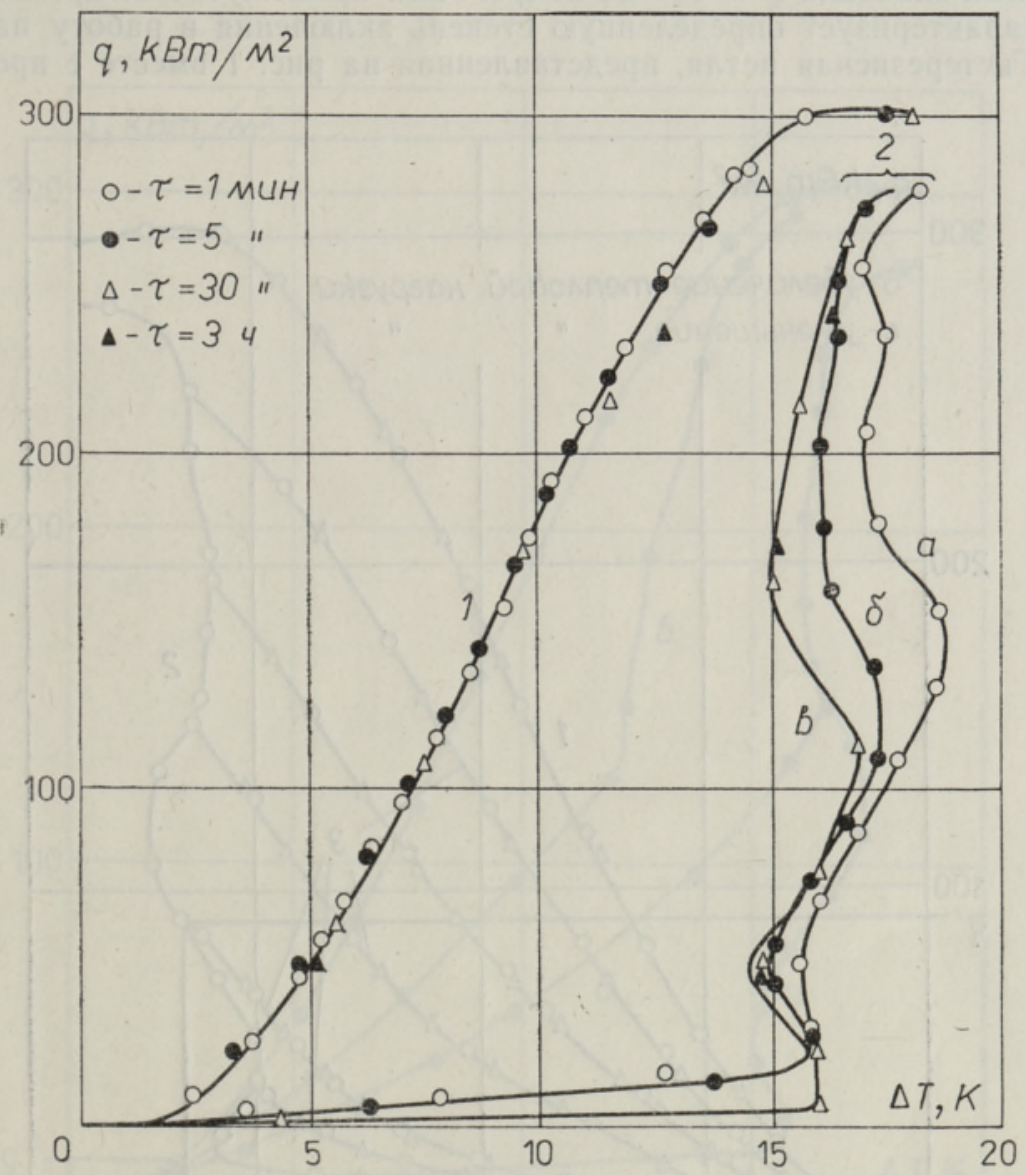

Рис. 1. Гистерезисные явления при кипении фреона-113 на алюминиевой поверхности с пористым бронзовым покрытием.

1 - кривая кипения после возвращения из пленочного режима, 2 - кривые кипения $(a, 6,8)$ на дегазированной поверхности при повышении тепловой нагрузки. Промежуточные положения восходящей ветви 2 при времени стабилизации: $a-1$ мин, б -5 мин, в -30 мин. 
терес переходные процессы в пределах гистерезисной петли при изменении плотности теплового потока.

Для экспериментального исследования было выбрано покрытие с малыми минимальными размерами пор и большой неоднородностью их по длине. Экспериментальная установка и методика проведения испытаний соответствуют данным, приведенным в $\left[{ }^{1}\right]$, т. е. мы использовали независимое изменение плотности теплового потока при кипении фреона-113 на горизонтальной поверхности диаметром 22 мм.

Устойчивость ветвей кривой кипения исследовалась при различной продолжительности выдержки постоянного теплового потока (рис. 1; $\Delta \tau=1,5,30$ мин и 3 ч). Левая ветвь гистерезисной петли весьма устойчивая, однако процесс кипения идет по этой ветви только после возвращения из кризиса или же при вскипании на недегазированной поверхности. Правая ветвь гистерезисной петли менее устойчива и со временем приближается к левой. Время стабилизации его положения для данного покрытия составляет примерно 1 ч. Особенностью правой ветви кривой кипения является то обстоятельство, что она появляется только при повышении плотности теплового потока. Уменьшение плотности теплового потока в любой точке $q^{\prime} \Delta T^{\prime}$ правой ветви дает новую стабильную промежуточную кривую кипения, на которой процесс кипения наблюдается до малых значений $q^{\prime \prime} \approx 10-20$ кВт/м². Эта промежуточная кривая кипения характеризует определенную степень включения в работу паровых пор. Гистерезисная петля, представленная на рис. 1 вместе с промежу-

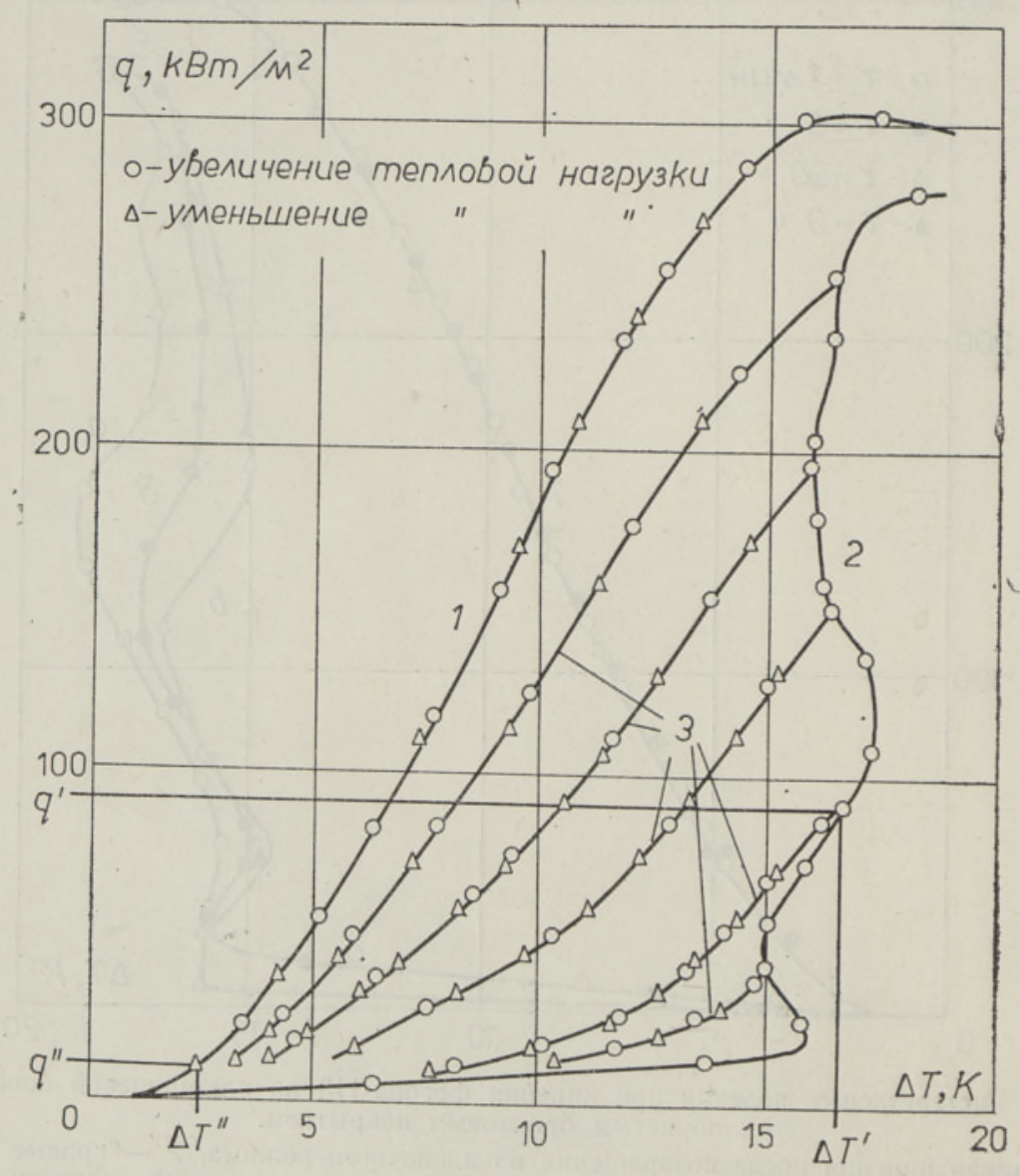

Рис. 2. Переходные кривые кипения (3) при различной степени открытия пор (1 и 2 см. на рнс. 1). 
точными кривыми при времени выдержки в каждой точке $\Delta \tau=5$ мин как при повышении, так и при понижении плотности теплового потока, показана на рис. 2. При этом кривые понижения и повышения плотности теплового потока практически совпадают, и их положение зависит только от начального состояния, т. е. от выбора точки $q^{\prime} \Delta T^{\prime}$, которая определяет степень открытия пор.

Кроме того, при плотностях теплового потока $q<q^{\prime \prime}$ происходит изменение состояния пористого покрытия - закрытие части паровых пор жидкостью. Представленные две промежуточные кривые кипения (рис. 3) получены при понижении плотности теплового потока до низких значений с последующим ее повышением $(\Delta \tau=5$ мин). Кривая 6 (рис. 3) качественно согласуется с промежуточными кривыми рис. 2, однако кривая $в$ (рис. 3 ) существенно отличается от них. Это свидетельствует о необратимости процессов закрытия и открытия паровых пор. Причиной этому служит, по-видимому, различие в законе распределения пор по размерам - по их минимальным (определяет открытие поры) и максимальным (закрытие) сечениям.

В связи со сложным поведением кривой кипения при наличии гистерезиса представляет интерес вопрос о механизме открытия паровых пор (включение активных центров в работу). Обычно считается, что при

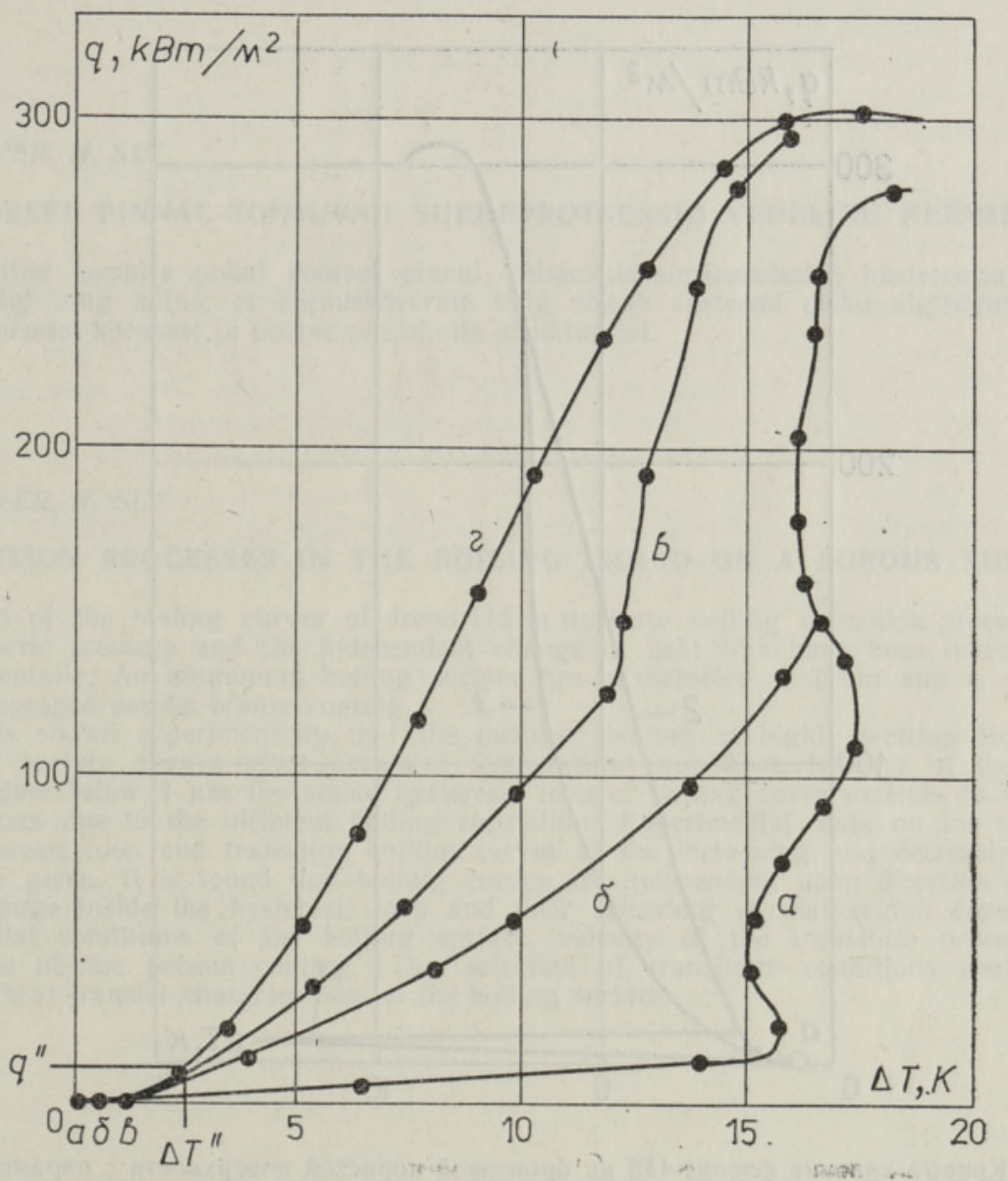

Рис. 3. Переходные крнвые кипения $(a, 6,8)$ в зависимости от начального состояния покрытия (2 - кривая кипения после возвращения из пленочного режима). 
кипении на поверхности без покрытия центры парообразования активизируются согласно неравенству

$$
a>2 T^{\prime \prime} \sigma / r_{\mathrm{Q}} \Delta T,
$$

где $a$ - радиус парового зародыша, $T^{\prime \prime}-$ температура насыщения, $\delta$ - коэффициент поверхностного натяжения, $r$ - скрытая теплота парообразования, @ - плотность пара, $\Delta T-$ перегрев жидкости относительно температуры насыщения.

Однако при наличии устойчивой гистерезисной петли температура поверхности при повышении плотности теплового потока не растет монотонно, а в некоторых случаях даже убывает монотонно. Тем не менее число открытых пор растет.

По-видимому, в данном случае основную роль играют пульсации давления, возникающие в пористом покрытии при течении через него жидкости и пара. Это ведет к локальному изменению температуры насыщения, что, согласно формуле (1), может быть причиной активации центра парообразования независимо от температуры поверхности.

Увеличение размеров пор позволяет ослабить гистерезисный характер кипения. Исследуем для этого, например, бронзовое пористое покрытие толщиной 0,12 мм, пористостью $44 \%$ и со средним размером пор 4,0 мкм (рис. 4). На кривой кипения отмечается только гистерезисная петля вскипания 1 , затем процесс идет скачком на устойчивую кривую

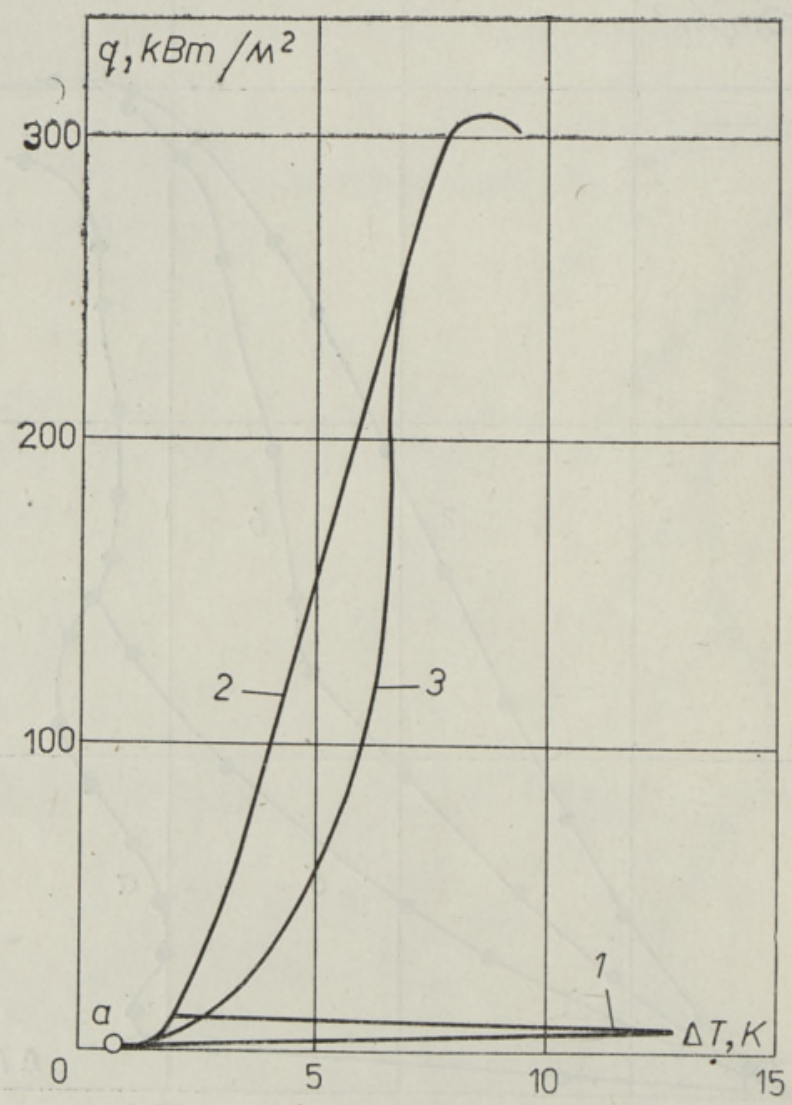

Рис. 4. Кривые кипения фреона-113 на бронзовой пористой поверхности с параметрами: $L=0,12$ мм, $\varepsilon=44 \%, \bar{a}=4,0$ мкм. 1 - петля вскипания, 2 - кривая кипения жидкости после вскипания, 3 - устойчивая 
кипения 2. Однако более детальное исследование показало, что и на этом покрытии можно получить прямую петлю гистерезиса путем понижения тепловой нагрузки (перегрева поверхности) до достаточно низких значений (точка $a$ на рис. 4) с последующим повышением тепловой нагрузки. Это соответствует промежуточным восходящим ветвям кривой кипения на рис. 3. В таком случае перегрев греющей поверхно-

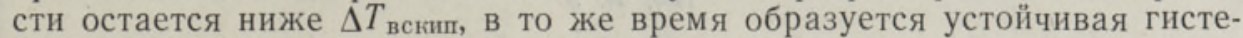
резисная петля 3. Ее ширина зависит от положения начальной точки роста тепловой нагрузки.

Учет выявленных закономерностей переходных процессов при кипении жидкости на пористой поверхности позволяет изменять теплообменные характеристики поверхности в желаемом направлении путем соответствующего временного изменения тепловой нагрузки.

\section{ЛИТЕ РА Т Р Р}

1. Техвер Я., Суй Х., Лянэ Р. Изв. АН ЭССР. Физ. Матем., 30, № 4, 376-380 (1981).

2. Техвер Я., Суй Х. Изв. АН СССР. Энерг. и трансп., № 4, 163-166 (1984). Ннститут термофизики и электрофизики
Академии наук Эстонской ССР
Поступила в редакцию 22/V 1984

\section{J. TEHVER, H. SUI}

\section{POORSEL PINNAL TOIMUVAD SIIRDEPROTSESSID VEDELIKU KEEMISEL}

On uuritud keemise puhul poorsel pinnal toimuvaid siirdeprotsesse hüstereesinähtuste olemasolul ning leitud, et keemiskõverate kulg sõltub süsteemi oleku algtingimustest, siirdeprotsessi kiirusest ja poorse pinnakatte struktuurist.

\section{J. TEHVER, H. SUI}

\section{TRANSITION PROCESSES IN THE BOILING LIQUID ON A POROUS SURFACE}

The run of the boiling curves of freon-113 in nucleate boiling transition processes at atmospheric pressure and the independent change of heat flux have been investigated experimentally. An aluminium boiling surface has a diameter of $22 \mathrm{~m}$ and is covered with a sprayed porous bronze coating.

It is shown experimentally that the incipient boiling of highly wetting liquid on degased surface always gives excessive temperature over-shoot. In case of the mean pore radius below $4 \mu \mathrm{m}$ the stable hysteresis loop of boiling curve extends to burnout heat fluxes due to the incipient boiling rectriction. Experimental data on the stability of hysteresis loop and transition boiling curves at the increasing and decreasing heat flux are given. It is found that boiling curves are independent upon direction of heat flux change inside the hysteresis loop and their behaviour in that region depends on the initial conditions of the boiling surface, velocity of the transition process and structure of the porous coating. The selection of transition conditions enables to change heat transfer characteristics of the boiling surface. 